\title{
Relationships Between Parenting Style, Self-Perception and Shyness Among Chinese Early Adolescents
}

\author{
Chen YingMin, Wu YunPeng, Zhang YuanJin, Wang Peng, Han Lei, Gao FengQiang \\ Shandong Normal University, Ji'nan, China
}

\begin{abstract}
The current study investigated the parenting style, self-perception, and shyness level of 1,338 junior high school students, so as to explore relationships between these variables. Results indicated that (1) The levels of the unassuming shyness, shyness when facing negative evaluation are higher than other dimensions of shyness; (2) The acceptance/involvement and strictness/supervision are negatively related with shyness; the acceptance/participation and strictness/supervision are positively correlated with self-perception; self-perception was negatively related with shyness; and (3) Parents' acceptance/participation can indirectly affect shyness through the self-perception; parents' strictness/supervision can affect shyness directly and indirectly through the self-perception.
\end{abstract}

Keywords: shyness, parenting style, self-perception, early adolescents

\section{Introduction}

Shyness refers to wariness and anxiety in the face of social novelty and perceived social-evaluation in spite of a desire to interact socially (Coplan, Findlay, \& Nelson, 2004). Shyness is very common in interpersonal relationships. Even in countries like America, the percentage of shy individuals is up to $40 \%-50 \%$ (Henderson \& Zimbardo, 1998). However, in Asian countries like China and Japan, the incidence of shyness is even higher, which is close to $60 \%$ (Pilkonis \& Zimbardo, 1979). Generally speaking, moderate shyness is a normal reaction. But the higher level of shyness may have more negative influences on individual social interactions, social adaption, and development (Schwartz, Snidman, \& Kagan, 1999). As a result, western countries carried out systematic research on shyness since 1970s, Chinese researchers started to pay attention to this field since the end of the 20th century. Shyness studies have become a hot topic of psychological studies.

The shyness studies found that the shyness level has a trend to increase with the age. For instance, Zimbardo's survey with grade 4-6 pupils found that shy students account for $42 \%$ of samples; while this proportion increased to 54\% among grades 7-8 students (Zimbardo \& Radl, 1999). Similarly, studies also

\footnotetext{
* Acknowledgments: This research was funded by two projects of Humanities and Social Sciences of the Ministry of Education of China (12YJC190004, 12YJC190009), by project of National Social Science Foundation of China (13BSH061) and Science and Technology Development Plan Projects of Shandong Province (2013GSF11802), the Key Discipline Construction Program of 12th Five-year Plan of Shandong province (Developmental and Educational Psychology).

Chen YingMin, Ph.D., School of Psychology, Shandong Normal University.

Wu YunPeng, Ph.D. candidate, School of Psychology, Shandong Normal University.

Zhang YuanJin, M.A., School of Psychology, Shandong Normal University.

Wang Peng, Ph.D., School of Psychology, Shandong Normal University.

Han Lei, Ph.D., School of Psychology, Shandong Normal University.

Gao FengQiang (Correspondence author), Ph.D., School of Psychology, Shandong Normal University. Email: gaofq_11@163.com.
} 
reported that, compared with the elementary students, middle school students are more likely to describe themselves as being shy (Simmons \& Rosenberg, 1975). Junior high school students who are in early adolescents are experiencing a special development stage of life. During this period, the dramatic physical and psychological changes and the rapid development of self may lead to intensification of individual shyness. This may cause distress to many aspects of their life including academic, emotion, behavior, and interpersonal interaction. Therefore, it is of theoretical and practical significance to focus on the shyness of junior high school students, and explore its influencing factors.

Buss assumed that excessive attention from parents and negative self-evaluation in childhood lead some individuals to be shy (Crozier, 2001), which means that individual shyness is related to parenting styles and self-consciousness. On this basis, the present study is to investigate the relationship of the parenting styles, self-perception, and shyness.

Parenting styles, which are often referred as family rearing, are the collection of parenting attitudes, behaviors, and non-verbal expression. It reflects the characteristics of parent-child interaction and shows cross-situational stability (Darling \& Steinberg, 1993). Parenting styles have important influence on individual growth. With specific reference to shyness, many studies have found the close relationship between shyness and parenting styles. Typically, authoritative parents often refuse or deny their child, punish their child severely, excessively interfere or overprotect their children. These practices often lead to or exacerbate child's shyness (Bešić \& Kerr, 2011; Mills \& Rubin, 1998). However, can the conclusions obtained in Western subjects be applied to Chinese subjects? This is a problem that the current study plans to explore.

Self-perception is the subjective evaluation of aspects of oneself (Harter, 1982), and it is the cognitive component of self-awareness. Studies have shown that there is a close relationship between self-consciousness and shyness. According to the reasons of shyness or the contexts that induce shyness, Buss (1986) classified shyness into fearful shyness and self-consciousness shyness. Fearful shyness is a kind of shyness which mainly pointed to strange and novel stimuli (including people and situations), which does not involve self-awareness activities. The self-consciousness shyness refers to the shyness one experiences when going through public attention, supervision, or evaluation. This kind of shyness is directly related with self-consciousness. The researchers also found that fearful shyness appeared very early, and it may appear in two months after birth; whereas self-consciousness shyness appeared fairly late, usually three to six years old, and it did not emerge until the development of self-consciousness. Previous study also found that young children associated shyness with worries about meeting strangers, while the older children associated shyness with being evaluated (Crozier \& Burnham, 1990). Experiencing rapid development of self-consciousness may be a critical factor for high prevalence of shyness during early adolescents.

In addition, many studies have found that parenting styles are closely related to self-perception. Studies indicated that authoritative parenting styles were predictive of higher academic competence. Meanwhile, authoritarian and permissive parenting styles were predictive of lower self-reliance (Kim \& Chung, 2003). Studies in China also found the relationship between parenting style and the self-concept in junior high school students (Zhang, 2004). Based on previous studies, the current study intends to focus on Chinese junior high school students, so as to explore (1) the general features of shy status of Chinese teenagers; and (2) the relationship between parenting, self-perception and shyness among early adolescents. We hypothesized that (1) The strictness/supervision of parenting is positively associated with shyness, and the acceptance/involve is negatively associated with shyness; (2) Parenting is positively associated with self-perception; and (3) There is 
a significant positive correlation between self-perception and shyness. Figure 1 shows the hypothesized structural equation model.

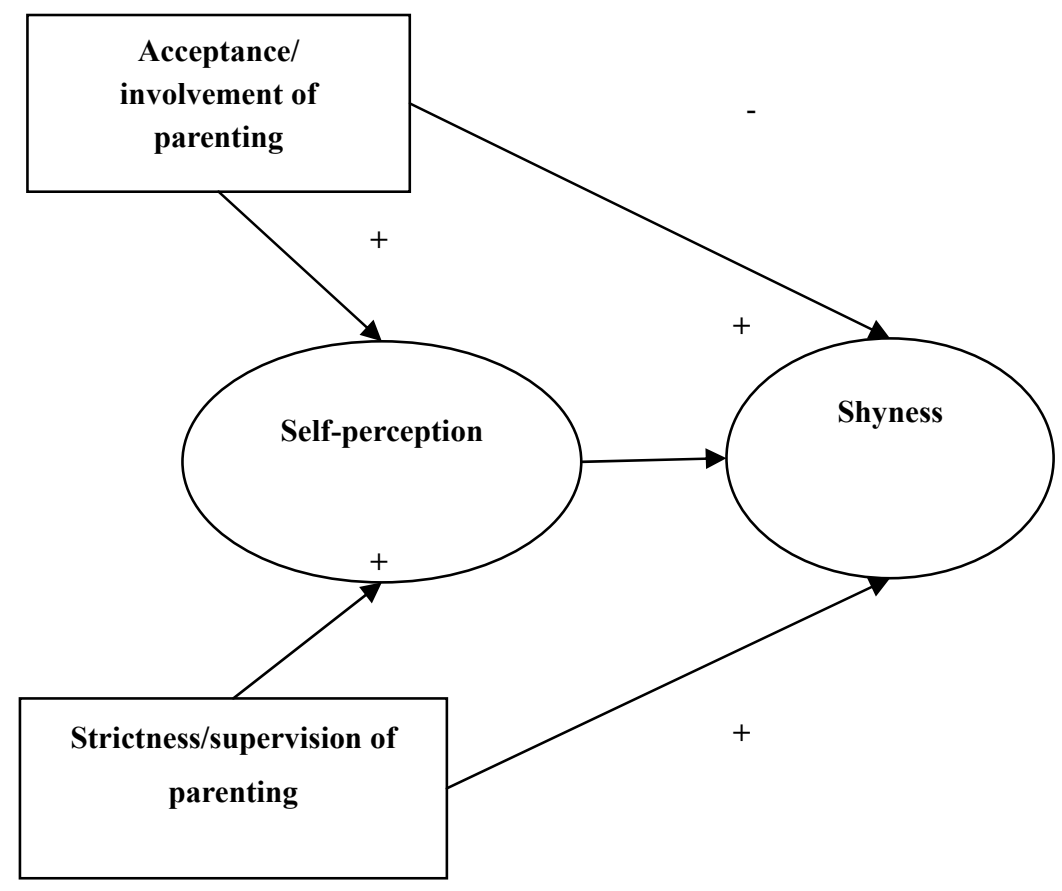

Figure 1. Hypothesized structural equation model of variables.

\section{Method}

\section{Participants}

The participants were selected from three junior high schools from Weifang City, Shandong Province, China by the cluster random sampling. There were 1,388 students ( 685 boys and 644 girls) from classes of Grades 7, 8, and 9. The age range of children was 11-16 $(M=13.63)$.

\section{Measures}

Parenting style. Children's perceptions of their parents' parenting styles were measured by PS_C (the Chinese version of parenting style) measurement applied Steinberg, Lamborn, Darling, Mounts, and Dornbusch (1994). This scale included three dimensions, acceptance/involvement, strictness/supervision, and psychological autonomy granting. The Chinese version had 26 items and used 5-point scale. In current study, we used the acceptance/involvement and strictness/supervision dimensions. Acceptance/involvement refers to the evaluation of children for their parents at reactivity, unassuming and participatory. The higher score means that it is easier for parents to accept and support children's requirements. Strictness/supervision refers to the evaluation of children for their parental supervision and perception of conduct regulation. Higher score means higher expectations and demands of parents for their children's mature and rational behavior. The questionnaire had a high degree of reliability and validity and was widely used in parenting style. In current study, Cronbach's alphas for these two subscales were 0.729 and 0.623 .

Self-perception profile for children. Children's self-perception was measured by SPPC (the self-perception profile for children) that developed by Harter and his collegues (Muris, Meesters, \& Fijen, 2003). The Chinese version has 36 items and used 4-point scale. It includes six dimensions (six items for each 
dimension) which include scholastic competence, social acceptance, athletic competence, physical appearance, behavioral conduct, and general self-esteem. Higher score means higher levels of self-perception. In current study, Cronbach's alphas for these six subscales ranged from 0.68 to 0.80 , with 0.808 for the whole scale.

Child shyness. Children were asked to complete a 31-item self-reported shyness scale for junior high school students (Chen, in press). This scale includes five subscales. Shyness for self-presentation ( 7 items) reflected the features of tension and back when they were in public. They were ashamed to express themselves with attention. Shyness towards stranger (6 items) reflected the shyness performance when they faced with a stranger. Shyness when facing negative evaluation ( 6 items) reflected the shyness performance when subjected to negative evaluation. Shyness towards opposite sex ( 7 items) reflected the shyness performance when they faced with the opposite sex. Unassuming shyness (5 items) reflected humble, low-key, unassuming, easygoing and other qualities when they were in interpersonal communication. The child shyness used 4-point scale, the higher scores mean higher degree of shyness. In the present study, the Cronbach's alphas for these five subscales ranged from 0.62 to 0.81 , with 0.90 for the whole scale.

\section{Data Analysis}

Data analysis of statistics and structural variance model were conducted by SPSS 17.0 and AMOS 7.0.

\section{Results}

\section{General Features of Shyness and Its Demographic Differences}

Table 1 shows the total shyness score and scores for each dimension of shyness. The average score of 31 items was 2.68. With regards to the level of these five dimensions, the comparison indicated the following order as unassuming shyness $>$ shyness when facing negative evaluation $>$ shyness towards opposite sex $>$ shyness towards stranger $>$ shyness for self-presentation.

Table 1

Descriptive Features of Shyness in Early Adolescents $(M \pm S D)$

\begin{tabular}{lrllllll}
\hline & \multicolumn{1}{l}{$N$} & sSP & sSt & sNE & sOS & Uns & Total score \\
\hline Grade 7 & 493 & $16.54 \pm 6.20$ & $13.67 \pm 5.31$ & $18.21 \pm 5.43$ & $17.15 \pm 6.48$ & $17.28 \pm 3.78$ & $82.88 \pm 19.95$ \\
Grade 8 & 381 & $16.22 \pm 6.00$ & $13.71 \pm 5.70$ & $18.82 \pm 5.63$ & $16.12 \pm 6.13$ & $17.36 \pm 4.05$ & $82.25 \pm 20.40$ \\
Grade 9 & 464 & $16.49 \pm 5.93$ & $13.94 \pm 5.97$ & $18.92 \pm 5.70$ & $17.01 \pm 6.62$ & $18.10 \pm 3.77$ & $84.48 \pm 21.86$ \\
Boy & 685 & $16.49 \pm 6.10$ & $13.47 \pm 5.55$ & $17.46 \pm 5.51$ & $17.01 \pm 6.54$ & $17.23 \pm 4.09$ & $81.67 \pm 20.85$ \\
Girl & 644 & $16.35 \pm 6.01$ & $14.09 \pm 5.77$ & $19.89 \pm 5.41$ & $16.57 \pm 6.33$ & $17.98 \pm 3.58$ & $84.90 \pm 20.63$ \\
Total & 1,338 & $16.43 \pm 6.04$ & $13.78 \pm 5.66$ & $18.63 \pm 5.59$ & $16.81 \pm 6.44$ & $17.59 \pm 3.87$ & $83.25 \pm 20.76$ \\
\hline
\end{tabular}

Note: $\mathrm{sSP}=$ Shyness for self-presentation, $\mathrm{sSt}=$ Shyness towards stranger, $\mathrm{sNE}=$ Shyness when facing negative evaluation, $\mathrm{sOS}$

= Shyness towards opposite sex, Uns = Unassuming shyness.

The total shyness score and scores for each dimension of shyness were used as dependent variables in the three grades (grade 7, grade 8, and grade 9) $\times 2$ (gender: boy, girl) MANOVA (multivariate analysis of variance).

The main effects of grade on shyness towards opposite $\operatorname{sex}\left(F(2,1308)=3.01, p<0.05\right.$, Partial $\eta^{2}=$ $0.005)$, and unassuming shyness $\left(F(2,1308)=5.87, p<0.01\right.$, Partial $\left.\eta^{2}=0.009\right)$, were significant. Post-hoc test illustrated that shyness towards opposite sex showed a significant difference between grade 7 and grade 8 students. In terms of unassuming shyness, significant differences were found between grade 7 and grade 9 ( $p$ $<0.001)$, and that between grade 8 and grade $9(p<0.001)$. 
The main effects of gender on shyness towards stranger $\left(F(1,1308)=4.13, p<0.05\right.$, Partial $\left.\eta^{2}=0.003\right)$, shyness when facing negative evaluation $\left(F(1,1308)=65.57, p<0.001\right.$, Partial $\left.\eta^{2}=0.047\right)$, unassuming shyness $\left(F(1,1308)=12.22, p<0.001\right.$, Partial $\left.\eta^{2}=0.009\right)$, and the total shyness score $(F(1,1308)=7.89, p<$ 0.01 , Partial $\eta^{2}=0.006$ ), were significant. All these differences indicated that girls showed higher shyness levels than boys.

Two-way interaction effects were neither significant in the total shyness score nor scores for each dimension of shyness.

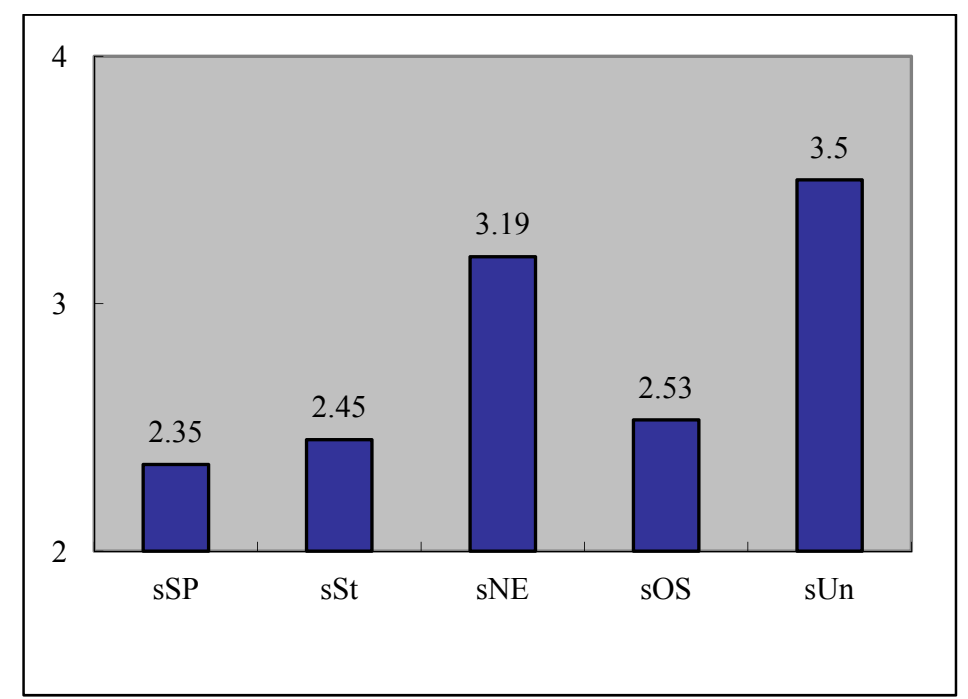

Figure 2. The bar graph for item average score in five dimensions of shyness.

\section{Correlations Between Parenting Style, Self-perception, and Shyness}

The correlation matrix was presented in Table 3. As results indicated that the acceptance/involvement and strictness/supervision were negatively and significantly related with shyness, and positively and significantly related with self-perception. All dimensions of self-perception were positively and significantly related with shyness.

Table 2

Correlation Matrix for Parenting Style, Self-perception, and Shyness

\begin{tabular}{|c|c|c|c|c|c|c|c|c|c|}
\hline & 1 & 2 & 3 & 4 & 5 & 6 & 7 & 8 & 9 \\
\hline 1. Scholastic competence & - & & & & & & & & \\
\hline 2. Social acceptance & $0.674^{* *}$ & - & & & & & & & \\
\hline 3. Athletic competence & $0.491^{* *}$ & $0.432^{* *}$ & - & & & & & & \\
\hline 4. Physical appearance & $0.573^{* *}$ & $0.523^{* *}$ & $0.523^{* *}$ & - & & & & & \\
\hline 5. Behavioral conduct & $0.627^{* *}$ & $0.529^{* *}$ & $0.373^{* *}$ & $0.510^{* *}$ & - & & & & \\
\hline 6. General self-esteem & $0.636^{* *}$ & $0.605^{* *}$ & $0.434^{* *}$ & $0.652^{* *}$ & $0.572^{* *}$ & - & & & \\
\hline 7. Acceptance/involvement & $0.376^{* *}$ & $0.342^{* *}$ & $0.191^{* *}$ & $0.303^{* *}$ & $0.350^{* *}$ & $0.372^{* *}$ & - & & \\
\hline 8. Strictness/supervision & $0.283^{* *}$ & $0.201^{* *}$ & $0.155^{* *}$ & $0.176^{* *}$ & $0.233^{* *}$ & $0.249^{* *}$ & $0.092^{*}$ & - & \\
\hline 9. Shyness & $-0.481^{* *}$ & $-0.482^{* *}$ & $-0.436^{* *}$ & $-0.458^{* *}$ & $-0.275^{* *}$ & $-0.505^{* *}$ & $-0.202^{* *}$ & $-0.335^{* *}$ & \\
\hline
\end{tabular}

Notes: ${ }^{*} p<0.05 ;{ }^{* *} p<0.01 ;{ }^{* * *} p<0.001$.

\section{The Establishment of Structural Equation Model}

The modified model resulted in $\chi^{2} / d f(246.59 / 57)=4.32$, RMSEA $=0.07$, GFI $=0.93$, TLI $=0.93$, CFI $=$ 
0.95, IFI $=0.95$, indicating that the model had an acceptable fit with the data. Figure 2 shows the evaluated full structural equation model with standardized coefficients.

\section{Direct Effects}

The acceptance/involvement had a positive direct effect on self-perception $(\beta=0.42, p<0.001)$, but a non-significant positive direct effect on shyness $(\beta=0.07, p=0.112)$. The strictness/supervision had a positive direct effect on self-perception $(\beta=0.26, p<0.001)$, and a negative direct effect on shyness $(\beta=-0.17, p<$ $0.001)$. It should be noted that, the self-perception has a negative effect on shyness $(\beta=-0.59, p<0.001)$.

\section{Indirect Effects}

Though the acceptance/involvement was negatively and significantly related with shyness (see Table 3), the results of structural equation model (Figure 2) indicated that the effect of acceptance/involvement on shyness was not statistically significant $(\beta=0.07, p=0.112)$. This indicates that the acceptance/involvement affects the shyness of adolescent through self-perception. Moreover, strictness/supervision of parenting style can not only directly affect the level of shyness of adolescents, but also have an indirect influence on it through self-perception.

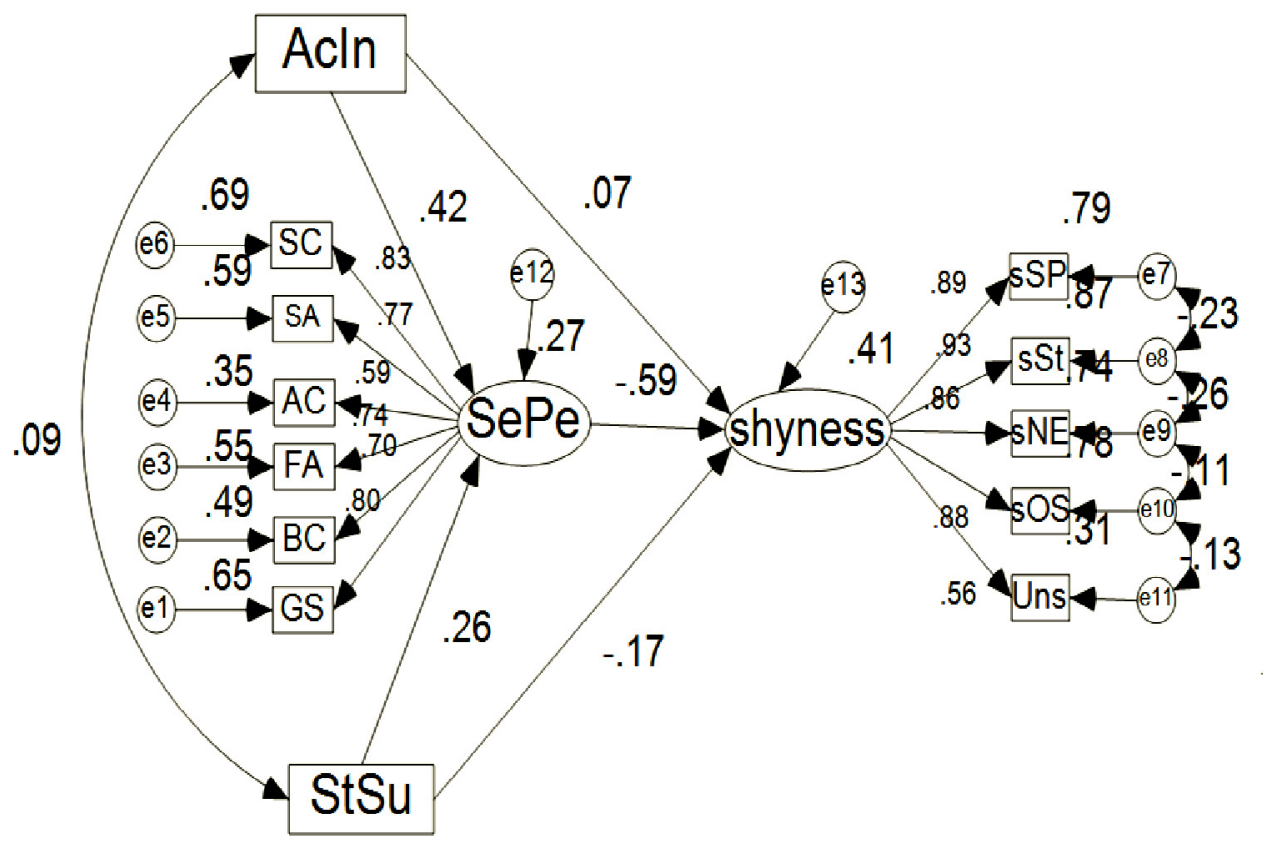

Figure 3. Modified structural equation model (Notes: $\mathrm{AcIn}=$ acceptance/involvement, $\mathrm{StSu}=$ strictness/supervision; $\mathrm{SePe}=$ self-perception, $\mathrm{SC}=$ scholastic competence, $\mathrm{SA}=$ social acceptance, $\mathrm{AC}=$ athletic competence, $\mathrm{FA}=$ physical appearance, $\mathrm{BC}=$ behavioral conduct, and $\mathrm{GS}=$ general self-esteem).

\section{Discussion}

In general, the level of shyness of Chinese adolescents is in the modest. This finding is consistent with other studies done in this field (Yuan, 2009; Zhang, 2011). Specifically, the levels of the unassuming shyness and shyness when facing negative are higher than other dimensions of shyness. This result can reflect the shyness features of early adolescents in the background of Chinese culture.

The finding that the dimension of unassuming shyness gets the highest score certified the influence of 
Chinese traditional culture. China is a collectivism-oriented country. The Chinese people hold the thought of being friendly while getting along with others, and always act with humility, present with a low profile, show an unassuming characteristic in interpersonal interactions, so as to avoid confliction and earn a positive evaluation. As a result, the prevalence of shyness level is relatively high in China. One possible reasons for this is the fact that, in China, the unassuming shyness is not considered as inappropriate, and in the contrast, it is even be considered as a merit that deserves praise and encouragement. In addition, shyness when facing negative evaluation is also higher. This result reflected that along with the growth of self-awareness, junior high school students become particularly sensitive to the evaluation, especially negative evaluation, of others. This may make it easier for them easier to become shy. This result supported the viewpoint of Buss (1986).

Consistent with previous studies (Yuan, 2009), the current study also found the tendency that shyness is increasing with age. This result indicated that, in China, the shyness level of individual may increase with age increase during early adolescent stage. Unsurprisingly, Chinese early adolescent girls showed higher level of shyness than boys in current study. Similar findings have been found in studies with the population of pre-school children (Y. Wu, J. Wu, Chen, L. Han, P. Han, Wang, \& Gao, 2014) and primary school children (Wu, Chen, \& Gao, 2013) in China.

The acceptance/involvement and strictness/supervision can indirectly influence child shyness through self-perception. Both two dimensions of parenting related with higher self-perception, while self-perception was related with lower shyness. Meanwhile, it is somewhat surprising to note that the strictness/supervision had a negative effect on child shyness. It is inconsistency with results in western samples may result from culture differences. As researches indicated that, for Chinese and Asian American children, not for White Americans, an authoritarian parenting style was found to bring about more positive outcomes than an authoritative style (Steinberg et al., 1994). In the Chinese culture background, the term "guan" means to "govern" as well as to "love" (Chao, 1994). The attention and high requirement from parents may also guarantee children's opportunities for learning social skills and motivations to make improvements.

\section{References}

Bešić, N., \& Kerr, M. (2011). Shy adolescents' perceptions of parental overcontrol and emotional coldness: Examining bidirectional links. Merrill-Palmer Quarterly, 57(4), 375-401.

Buss, A. H. (1986). Two kinds of shyness. In Schwarzer (Ed.), Self-related cognition in anxiety and motivation (pp. 65-75). Hillsdale, N.J.: Erlbaum.

Chao, R. K. (1994). Beyond parental control and authoritarian parenting style: Understanding Chinese parenting through the cultural notion of training. Child Development, 65(4), 1111-1119.

Chen, Y. (in press). Development of shyness scale for Chinese junior high school students. Journal of Shandong Normal University (Natural Science).

Coplan, R. J., Findlay, L. C., \& Nelson, L. J. (2004). Characteristics of preschoolers with lower perceived competence. Journal of Abnormal Child Psychology, 32(4), 399-408.

Crozier, W. R. (2001). Understanding shyness: Psychological perspectives. Palgrave Macmillan.

Crozier, W. R., \& Burnham, M. (1990). Age-related differences in children's understanding of shyness: Age-related differences in children's understanding of shyness. British Journal of Developmental Psychology, 8, 179-185.

Darling, N., \& Steinberg, L. (1993). Parenting style as content: An integrative modle. Developmental Psychological, 113, 487-496.

Harter, S. (1982). The perceived competence scale for children. Child Development, 53, 87-97.

Henderson, L., \& Zimbardo, P. (1998). Shyness: Encyclopedia of mental health. San Diego: Academic Press.

Kim, H., \& Chung, R. H. G. (2003). Relationship of recalled parenting style to self-perception in Korean American college students. The Journal of Genetic Psychology, 164(4), 481-492. 
Mills, R. S. L., \& Rubin, K. H. (1998). Are behavioral and psychological control both differentially associated with childhood aggression and social withdrawal? Canadian Journal of Behavioral Science, 30, 132-136.

Muris, P., Meesters, C., \& Fijen, P. (2003). The self-perception profile for children: Further evidence for its factor structure, reliability, and validity. Personality and Individual Differences, 35(8), 1791-1802. doi:10.1016/S0191-8869(03)00004-7

Pilkonis, P. A., \& Zimbardo, P. G. (1979). The personal and social dynamics of shyness. In Emotions in personality and psychopathology (pp. 131-160). US: Springer.

Schwartz, C. E., Snidman, N., \& Kagan, J. (1999). Adolescent social anxiety as an outcome of inhibited temperament in childhood. Journal of the American Academy of Child and Adolescent Psychiatry, 38(8), 1008-1015.

Simmons, R. G., \& Rosenberg, F. (1975). Sex, sex roles, and self-image. Journal of Youth and Adolescence, 4, 229-258.

Steinberg, L., Lamborn, S. D., Darling, N., Mounts, N. S., \& Dornbusch, S. M. (1994). Over-time changes in adjustment and competence among adolescents from authoritative, authoritarian, indulgent, and neglectful families. Child Development, 65(3), 754-770.

Wu, Y., Chen, Y., \& Gao, F. Q. (2013). The development and application of self-reported shyness scale for primary school children. China Journal of Health Psychology (in Chinese), 21(11), 1698-1701.

Wu, Y., Wu, J., Chen, Y., Han, L., Han, P., Wang, P., \& Gao, F. (2014). Shyness and school adjustment among Chinese preschool children: Examining the moderating effect of gender and teacher-child relationship. Early Education \& Development.

Yuan, B. (2009). The characteristics of shyness and its relationships with self-efficiency and subjective happiness among middle school students. Shan'xi Normal University.

Zhang, D. (2011). The development and application of middle school student shyness scale. Shandong Normal University.

Zhang, X. (2004). The relationship between self-concept and parenting style among junior high school students. Chinese Journal of School Health, 25(1), 82-83.

Zimbardo, P. G., \& Radl, S. L. (1999). The shy child (reprinted). Los Altos, C.A.: Malor Press. 